\title{
Supporting Information: Pulsed Force Kelvin Probe Force Microscopy
}

\author{
Devon S. Jakob ${ }^{1}$ Haomin Wang, ${ }^{1}$ and Xiaoji G. $\mathrm{Xu}^{1}$ \\ ${ }^{1}$ Department of Chemistry, Lehigh University, 6 E Packer Ave. Bethlehem, PA, 18015, United States \\ Corresponding Email: xgx214@ lehigh.edu
}

\section{Supporting Information}

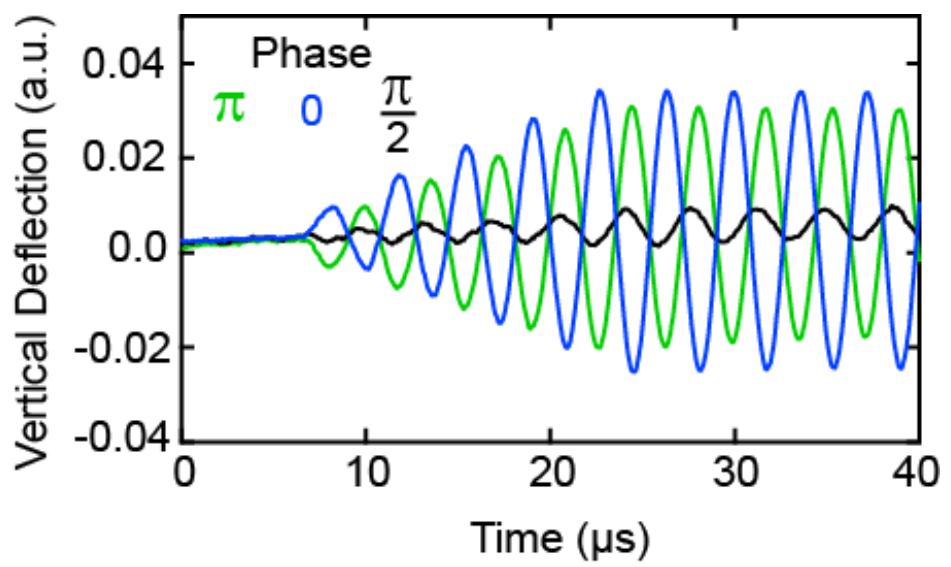

Figure S1. The cantilever deflection curves extracted while maintaining setpoints close to $\pi$ (green), 0 (blue), and $\pi / 2$ (black). As the phase approaches $\pi / 2$, the cantilever deflection due to the induced Kelvin force disappears and the value of CPD is revealed. This point corresponds to the crossing point of the two branches in the DC bias sweep shown in Fig. $3 b$ of the main text. When the DC bias voltage is not equal to the CPD, the cantilever oscillations are still present. Depending on the branch in which the negative feedback loop is operating on, the phase will be close to either $\pi$ or 0 . 

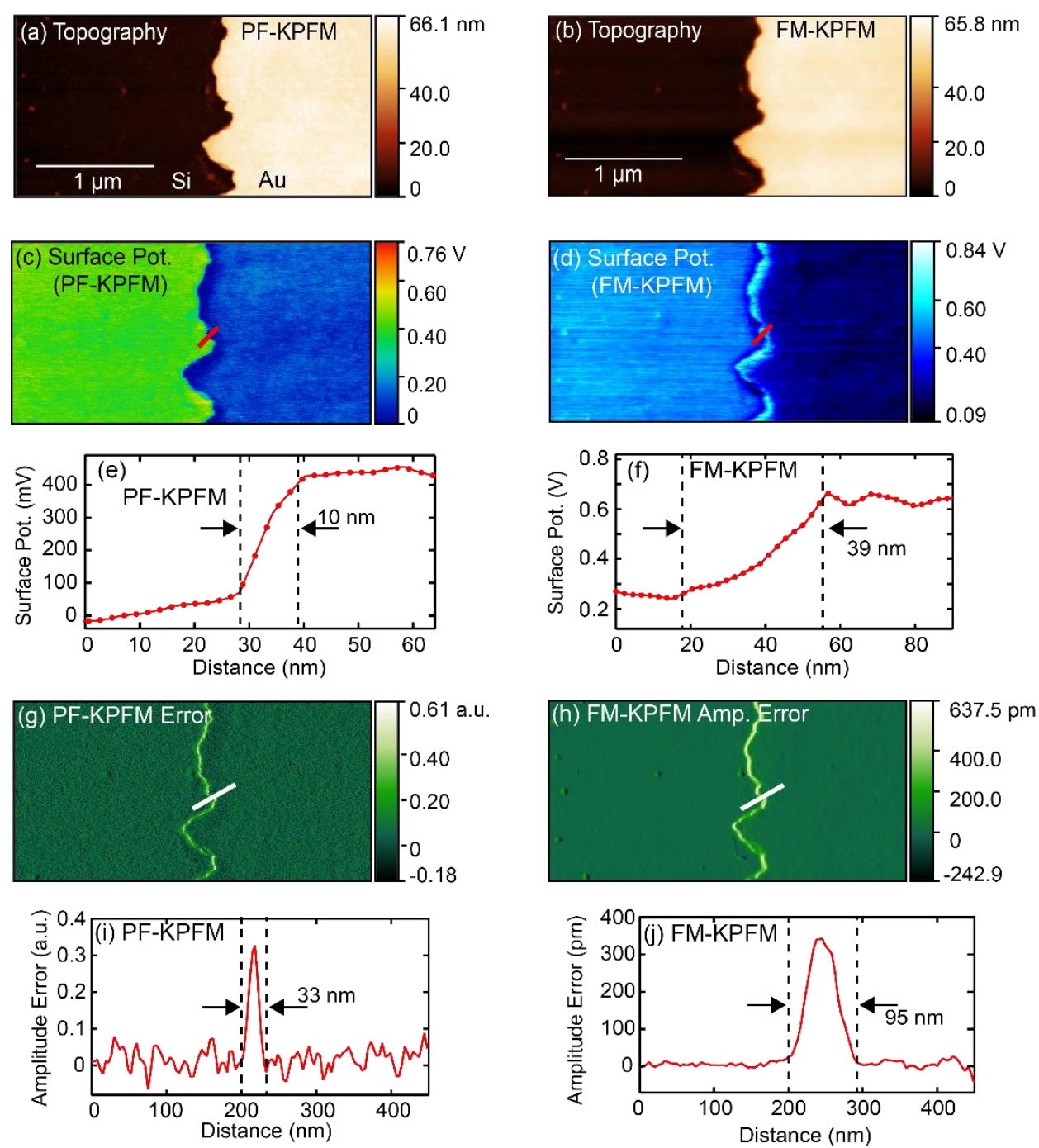

Figure S2. Comparison between PF-KPFM and FM-KPFM measurements on a standard KPFM calibration sample. (a) Topography of the sample obtained with PF-KPFM. The sample is composed of silicon on the left and gold on the right. The scale bar is $1 \mu \mathrm{m}$. (b) Topography of the same area obtained with FM-KPFM. (c) The surface potential of the sample measured with PFKPFM. This measurement is identical to Fig 5c of the main text. (d) The surface potential of the same area measured with FM-KPFM. (e) The spatial resolution of the PF-KPFM measurement is estimated to be $10 \mathrm{~nm}$, taken as the lateral width of the surface potential difference at the interface between silicon and gold, shown s the red line in (c). (f) The spatial resolution of the FM-KPFM measurement is estimated to be $39 \mathrm{~nm}$ over the same area. (g) The error map extracted from the PF-KPFM measurement. The error is extracted as the difference between the external setpoint and the measured oscillation amplitude of the cantilever. (h) The error map for FM-KPFM over the same area. (i) The lateral distance associated with the error extracted with PF-KPFM, taken from the cross section shown by a white line in (e) The lateral error width is $33 \mathrm{~nm}$. (j) The lateral width associated with the error for FM-KPFM, taken from the cross section shown by a white line in (f). The lateral error width for FM-KPFM is $95 \mathrm{~nm}$, suggesting a lower achievable spatial resolution 
for FM-KPFM compared to PF-KPFM. The type of AFM probe used is the same in both measurements (HQ:NSC15/Pt, Mikromasch)

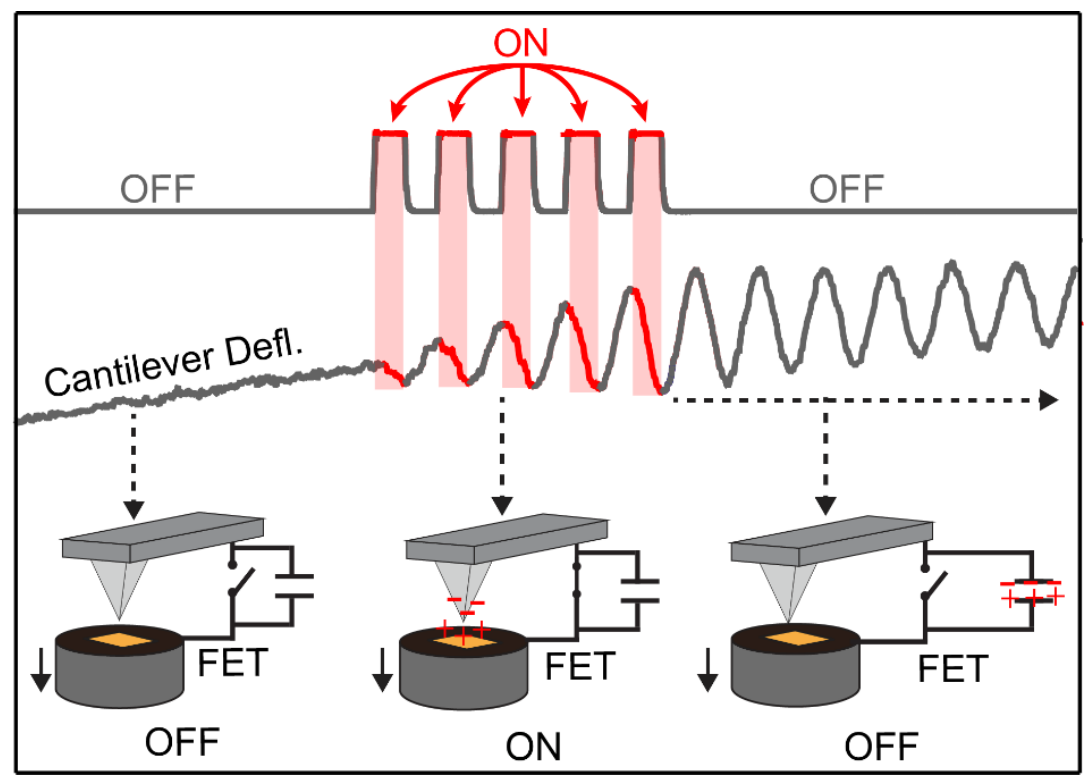

Figure S3. PF-KPFM signal generation schematic. Shortly after the AFM probe detaches from the sample, a voltage train is sent to the FET (top trace) to change it into the ON state. In the ON state, Coulombic attraction between AFM tip and sample induce cantilever oscillations at its resonant frequency (center trace). Since the duty cycle of the pulses is $50 \%$, each consecutive electrical connection allows for the induced oscillation to be resonantly enhanced by the previously induced oscillation(s). The timing of the ON states is indicated by the red portions of the FET and cantilever traces, in which the oscillation amplitude enhancement is seen. The charge migration is shown at the bottom of the schematic. After tip detachment, the FET is in its OFF state. During the ON states, charges are free to pass between tip and sample, causing Coulombic attraction. During the OFF state, the FET acts as a capacitor that is connected to the tip and sample. The much larger capacitance of the FET than the tip-sample junction means that practically all charges between the tip and the sample are now drawn to the FET residual capacitor. The Coulombic force disappears. 